\title{
Ultra-Wideband Microwave Imaging of Heterogeneities
}

\author{
Matthew Yedlin ${ }^{\mathrm{a}, \mathrm{b}, *}$, Anthony Cresp ${ }^{\mathrm{b}}$, Christian Pichot ${ }^{\mathrm{b}}$, \\ Ioannis Aliferis ${ }^{b}$, Jean-Yves Dauvignac ${ }^{\text {b }}$, Stéphane Gaffet ${ }^{\mathrm{c}}$, \\ Guy Sénéchal ${ }^{\mathrm{d}}$
}

${ }^{a}$ Department of Electrical and Computer Engineering, University of British Columbia, 2332 Main Mall, UBC Campus, Vancouver, Canada

${ }^{\mathrm{b}}$ Laboratoire d'Électronique, Antennes et Télécommunications, Université de Nice Sophia-Antipolis, CNRS ; 250, rue Albert Einstein, FR-06560 Valbonne, France

${ }^{\mathrm{c}}$ UMR Géosciences Azur, CNRS / UNSA / IRD / UPMC, Sophia - Antipolis, CNRS ; 250, rue Albert Einstein, FR-06560 Valbonne, France

${ }^{\mathrm{d}}$ UMR 5212, Modélisation et Imagerie en Géosciences, Pau IPRA - Université de Pau et des Pays de l'Adour, BP 1155, FR-64013 Pau Cedex, France

\begin{abstract}
The technique of time-reversal acoustics was applied to image a bottle filled with saline, using an eight element Vivaldi antenna array with frequency bandwidth 2 to $8 \mathrm{GHz}$. At these short length scales, a smooth three-dimensional image of the bottle was obtained, with the usual limitations imposed by limited offset and frequency. Time snapshots of the wavefield evolution in reversed time are presented for two real data sets. The first, shows the focusing for the single target of the bottle, while the second demonstrates the principle for two targets.
\end{abstract}

Key words: three-dimensional reverse-time imaging, ground penetrating radar, ultra-wideband imaging, migration

* Corresponding Author: Department of Electrical and Computer Engineering, University of British Columbia, 2332 Main Mall, Vancouver, B.C., Canada.

Tel.: 1604822 8236; fax: 16048225949

Email address: matty@ece.ubc.ca (Matthew Yedlin). 


\section{Introduction}

Considerable effort and expertise has been expended in using microwaves as an energy probe to obtain images of the interior of a target which is illuminated by such energy. There are two major areas of the use of microwaves for imaging target objects. One is the interior imaging of dielectric objects, via inverse scattering as applied to the well-known exterior scattering problem and the other is the employment of ground penetrating radar (GPR) for imaging the near surface of the earth as presented by numerous authors (Munk and Sheets, 1997; Sneddon et al., 2002; Johnson and Poeter, 2003; Yarovoy et al., 2003; Daniels, 2004).

In the field of inverse scattering as is utilized for dielectric imaging, fields of application encompass the detection of tumors in medicine (Miyakawa et al., 1999, 2004; Holmes et al., 2000) and non-destructive testing of concrete structures (Pastorino et al., 2001; Marklein et al., 2002; Caorsi et al., 2004). The principal algorithms used in these applications include diffraction tomography at $3 \mathrm{GHz}$ (Bolomey et al., 1982; Pichot et al., 1985) and non-linear inversion techniques introduced by Pichot et al. (2004).

In this paper we present the application of microwave imaging to the exterior scattering problem, in which we use ultra-wideband (UWB) electromagnetic radiation $(2$ to $8 \mathrm{GHz}$ ) to illuminate a target consisting of a bottle of water filled with saline, which has a dielectric constant with real and imaginary parts. The radiated energy is recorded by an eight element exponentially tapered slot antenna (ETSA) array. To obtain the image of the scattering target, instead of using traditional inverse scattering techniques, which are generally very computationally intensive, we employ the reverse-time techniques of Fink and co-workers (Fink et al., 2000; Derode et al., 2002; Fink, 2006). For the exterior scattering problem, the technique of Fink has a complete analogy with the principles used in reverse-time migration, (Baysal et al., 1983; Chang and McMechan, 1987, 1990; Wapenaar et al., 2005) in which the background scattering models employed have a correlation length that is comparable or larger than the propagation wavelength. The scattering data presented herein are very localized and well-suited for the reverse-time algorithms, though the images we obtain are not complete inversions yielding the dielectric structure of the scattering object. The reverse-time propagation of the single-target scattering data are presented as a snapshot sequence. For comparison, a twotarget snapshot sequence is also presented. 


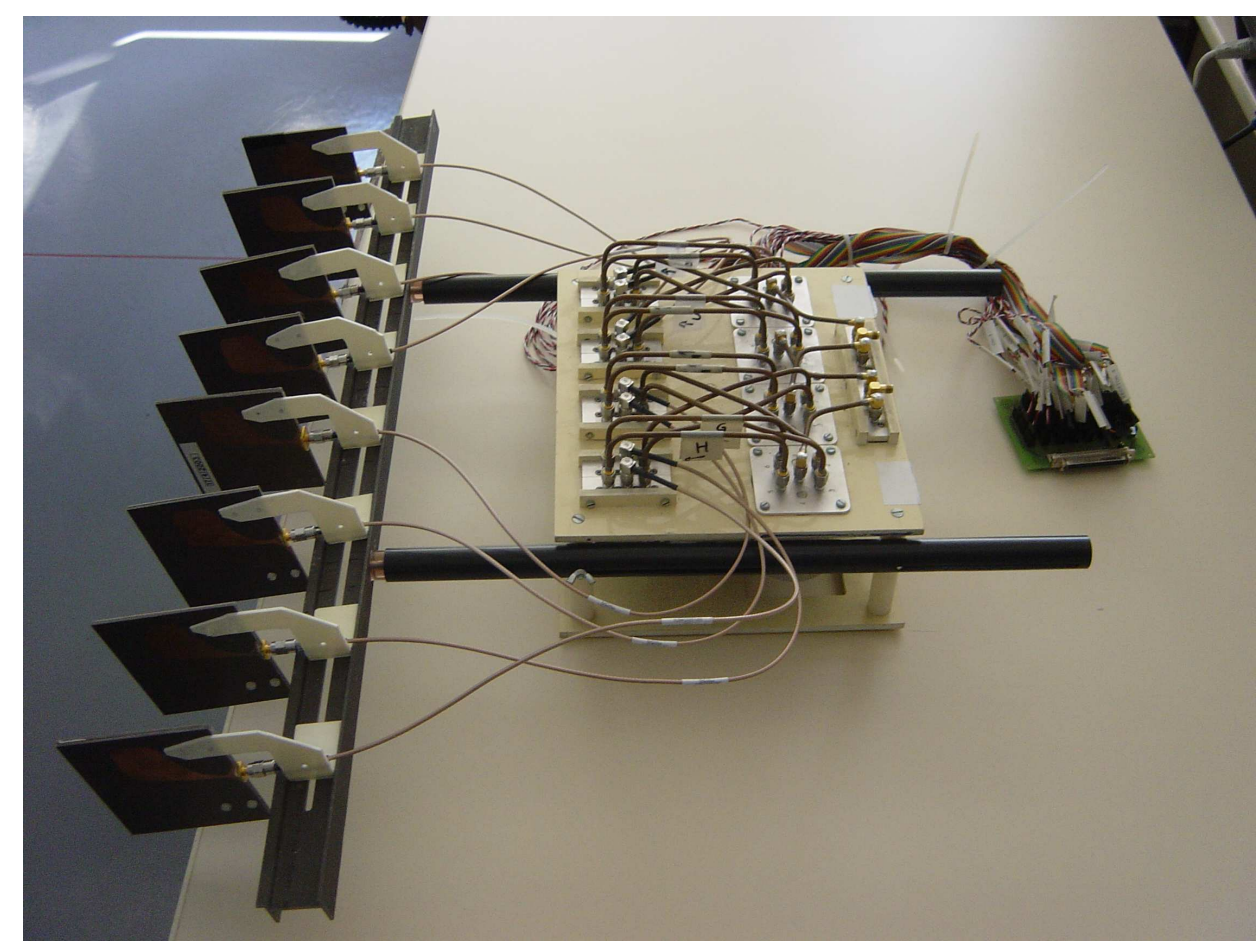

Fig. 1. View of the eight element exponentially tapered slot antenna array connected to an eight to one multiplexer

\section{Methods}

\subsection{Experimental Configuration for Ultra-Wideband Imaging}

For the scattering experiment described in this research, we used a linear eight element array (Figure 1) of symmetric antipodal antennas, members of the exponentially tapered slot class, also known as Vivaldi antennas (Gibson, 1979; Guillanton et al., 1998; Guillanton, 2000). Each exponentially tapered slot antenna (ETSA) was constructed on a Duroid substrate, with copper etching done using printed circuit technology. These antennas have a bandwidth from $1.4 \mathrm{GHz}$ to $20 \mathrm{GHz}$ with a reflection coefficient magnitude less than $-10 \mathrm{~dB}$ over the entire band and therefore, they fall into the category of Ultra-Wideband (UWB) antennas. Their gain at $5 \mathrm{GHz}$ is of the order of $7 \mathrm{~dB}$ and at $9.5 \mathrm{GHz}$ it is $10 \mathrm{~dB}$. A detailed study by Chatelée (2006) has demonstrated that the inter-antenna coupling does not degrade the performance significantly if radiation frequencies are considered above $2.5 \mathrm{GHz}$. 


\subsection{Data Acquisition}

The eight element array was deployed by sequentially using one antenna as a transmitter and then receiving scattered energy by the entire array, with array elements heretofore labeled by the letters $\mathrm{A}$ to $\mathrm{H}$. The entire array was driven by a two-port vector analyzer HP8720B with a bandwidth from .01 to $20 \mathrm{GHz}$ and a frequency resolution of $100 \mathrm{kHz}$. Since only two ports were available, an electromechanical multiplexer (Guillanton, 2000) was employed. For the scattering experiment the bandwidth chosen was 2 to $8 \mathrm{GHz}$ with frequency binning of $15 \mathrm{MHz}$.

Two sets of data were collected. First a background set of data was collected without the scattering target in place. Then a set of data was collected with the target in place. In the experimental setting considered, there was no interaction between the target scatterer and the background, enabling us to obtain the scattered electromagnetic field from the target via a simple subtraction of the two data sets. All data, collected in the frequency domain, was then inverse Fourier transformed to obtain the time response.

\subsection{Imaging Algorithm}

In the scattering experiments we describe in this research, an incident wave, generated by a transmitter, impinges on a target, which creates a scattered wave. The scattered wave is complicated, consisting of reflections, refractions, diffractions and surface waves. Our objective is to refocus the scattered wave created by the target and thus image the target, which is viewed as an effective ensemble of secondary sources. One of the principal accepted algorithms for this type of imaging is the application of generalized least squares methods which yields the target structure.

We have chosen an alternative method, a variant of that originally created by Fink (2006) and applied to the refocusing of recorded scalar wavefields back to the source of the recorded scalar wavefield. The Fink technique, which is extremely effective in imaging heterogeneous media, is based on reciprocity and can be decomposed into two basic steps. In the first step, data recorded by an array of receivers, is time-reversed. In the second step, the time-reversed data is retro-propagated from each receiver back into the medium in which the source is located. If the medium is non-attenuating then it is intuitively clear that the retro-propagated waves will constructively interfere at the source, yielding the focused source image. However, if the retro-propagation is performed on a computer, in which the velocity is an unknown parameter, the focus will have a distribution centered at the wrong location. In addition, if the 
array of receivers does not surround the source and is not sufficiently dense, the focus will be blurred.

Schematically, the time reverse algorithm is shown in Figure 2, in which two receivers obtain the scattered signal from a single transmitter. In Figure 2b, the scattered arrivals are presented in time-reversed fashion with $t^{\prime}=T-t$. The retro-propagated and summed signals are shown in Figure 2c. Here they interfere constructively at the target location defined by $t^{\prime}=T-\frac{r_{0}}{c}$. With the proper choice of the speed $c$, we note that the scattered signals constructively interfere at the right location in time.

Mathematically, in a kinematic context, the foregoing procedure can be described as follows. First define the point source signal leaving the source as $\delta(t)$. The signal leaving the scatterer is given by $\delta\left(t-\frac{r_{0}}{c}\right)$, while the one arriving at receiver 1 can be written as $\delta\left(t-\frac{r_{0}+r_{1}}{c}\right)$ and, in a similar way, the signal at receiver 2 is $\delta\left(t-\frac{r_{0}+r_{2}}{c}\right)$. Time-reversal corresponds to a change of variable: the new time variable, $t^{\prime}=T-t$, reverses the time and contains information on $T$, an appropriate stopping time.

In the example taken here, after time reversal, the source is located at time $t^{\prime}=T$, the scatterer at $t^{\prime}=T-\frac{r_{0}}{c}$, while arrivals at receivers 1,2 correspond to $t^{\prime}=T-\frac{r_{0}+r_{1}}{c}$ and $t^{\prime}=T-\frac{r_{0}+r_{2}}{c}$, respectively.

Upon propagation of the time-reversed pulses, the signal from each receiver is appropriately delayed, resulting to a convolution with a delta function. For receiver 1 , this operation gives:

$$
\delta\left(T-\left[t^{\prime}+\frac{r_{0}+r_{1}}{c}\right]\right) * \delta\left(t^{\prime}-\frac{r_{1}}{c}\right)=\delta\left(T-t^{\prime}-\frac{r_{0}}{c}\right)
$$

while for receiver 2 ,

$$
\delta\left(T-\left[t^{\prime}+\frac{r_{0}+r_{2}}{c}\right]\right) * \delta\left(t^{\prime}-\frac{r_{2}}{c}\right)=\delta\left(T-t^{\prime}-\frac{r_{0}}{c}\right)
$$

We obtain the image by summing up contributions like 1 and 2 .

Our algorithm is based on time-reversal followed by retro-propagation and is extended to consider vector wavefields. We assume that the scattered data originates from an ensemble of non-interacting point scatterers. We call these CSP's (Candidate Scattering Points). We model the radiated field by the CSP as a short dipole with each dipole having the same amplitude. The receivers are also modeled as short dipoles. In fact, the retro-propagation is done using the Green's function for the requisite wave propagation. Mathematically, we 


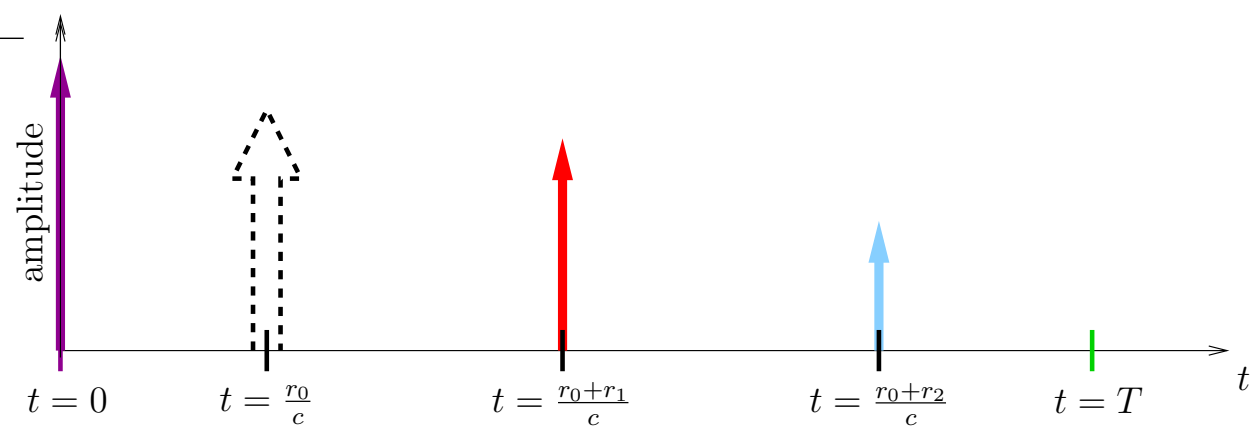

(a)

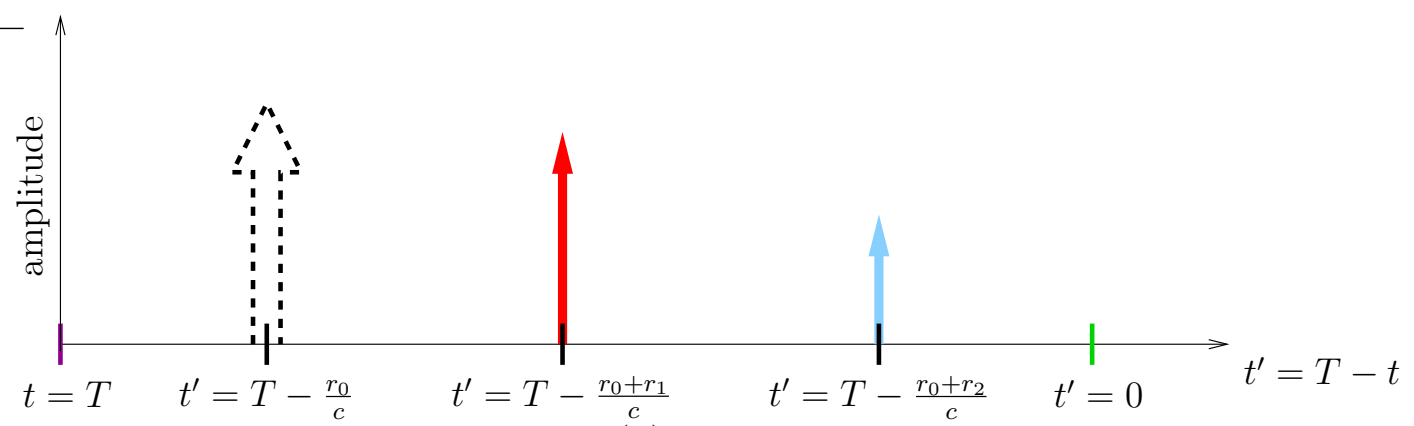

(b)

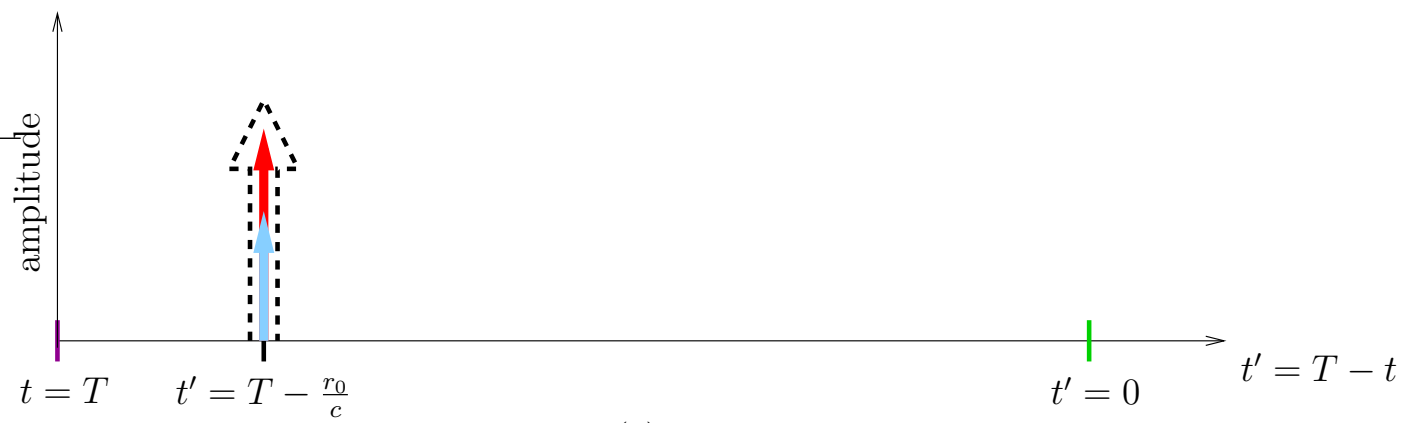

(c)

Fig. 2. (a) Schematic of a transmitted wave travelling a distance $r_{0}$ to the target and recorded at two receivers respectively located at a distance $r_{1}$ and $r_{2}$ from the target. Shown here is the complete idealized delta function time series - the transmitted data leaving the source, the arrival time at the scatterer and the arrival time at each receiver. b) Time-reversed version of a). c) Retropropagation of time-reversed data back to the scatterer with the resultant constructive interference at $t^{\prime}=T-\frac{r_{0}}{c}$.

write the frequency domain Green's function as

$$
\begin{aligned}
\mathbf{E}= & -\frac{\mathrm{j} Z_{0} I \mathrm{~d} l}{2 \pi k_{0}} \cos \theta\left(\frac{\mathrm{j} k_{0}}{r^{2}}+\frac{1}{r^{3}}\right) \mathrm{e}^{-\mathrm{j} k_{0} r} \mathbf{a}_{\mathbf{r}} \\
& +\frac{\mathrm{j} Z_{0} I \mathrm{~d} l}{4 \pi k_{0}} \sin \theta\left(-\frac{k_{0}^{2}}{r}+\frac{\mathrm{j} k_{0}}{r^{2}}+\frac{1}{r^{3}}\right) \mathrm{e}^{-\mathrm{j} k_{0} r} \mathbf{a}_{\theta}
\end{aligned}
$$

where $I$ is the dipole current, $\mathrm{d} l$ is the dipole length, $Z_{0}$ is the impedance of free space and $k_{0}$ is the free space wave number, which is assumed to be 
known.

We note that the Green's function can be rewritten in terms of impulse functions, their derivatives and integrals, so that the retro-propagation can be carried out efficiently. The algorithm for imaging used in this research is summarized below as follows:

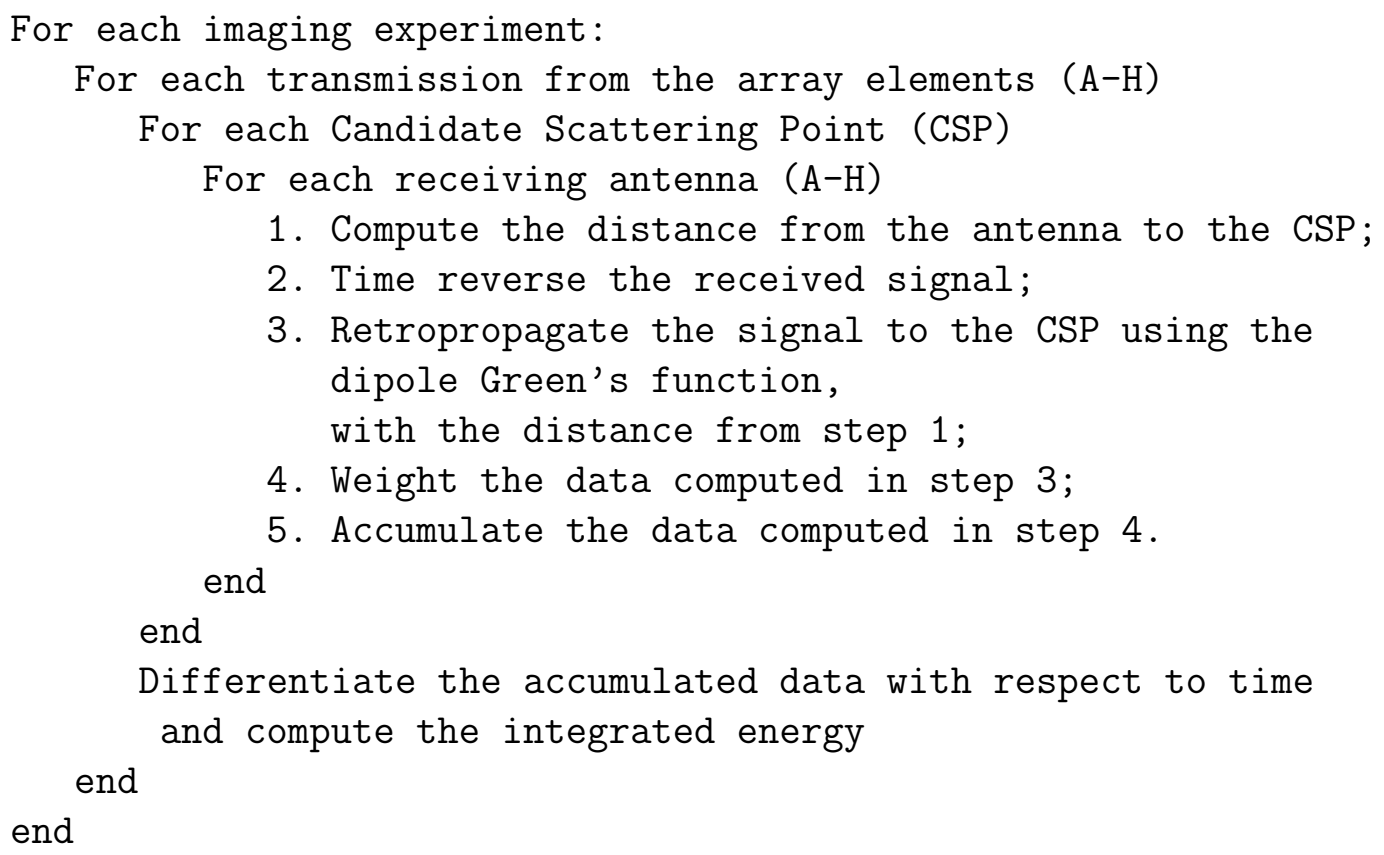

The foregoing description of the imaging algorithm assumes that the secondary source dipole and the receiving dipole lie in the same plane, in this case $z=0$. If we are imaging CSP's at $z \neq 0$, we must project the secondary dipole vector onto the receiving dipole, after doing the weighting in step 4.

\section{Results}

\subsection{Results for UWB Reverse-Time Imaging}

In this Section we present the results of using the reverse time imaging algorithm described above to the scattering data collected using the techniques described in Section 2.2. Figure 3 shows the experimental configuration of the array, with the target symmetrically positioned a distance $40 \mathrm{~cm}$ from the center of the array. The ETSA spacing is $5 \mathrm{~cm}$ with a total array length of $35 \mathrm{~cm}$. Given that the performance of the antenna elements is not significantly affected by mutual coupling in the frequency band of interest, 2 to $8 \mathrm{GHz}$, each element may be considered as an independent transmitter/receiver. For the 


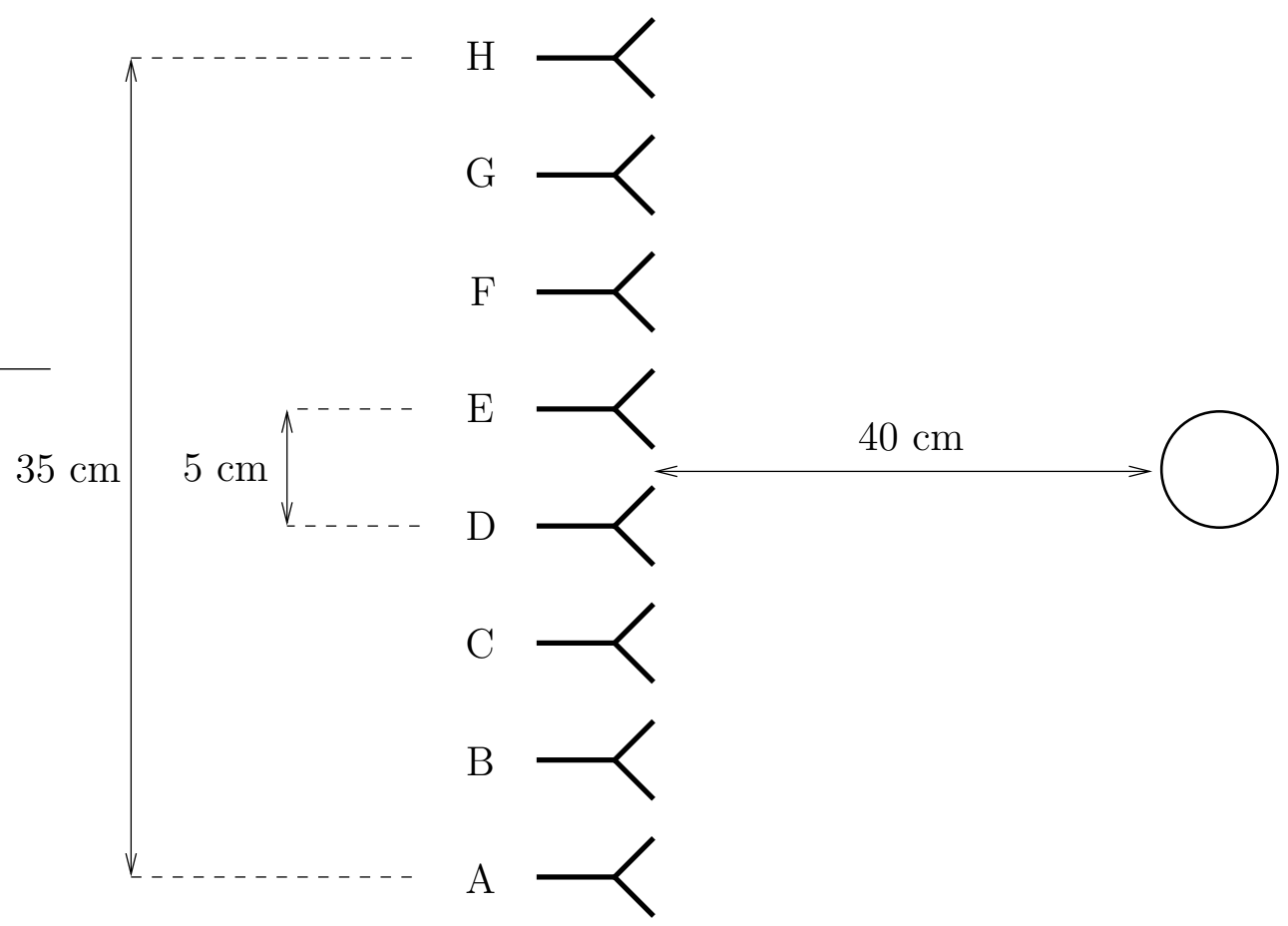

Fig. 3. Schematic of scattering experiment from the eight element Vivaldi antenna array. The target bottle is located $40 \mathrm{~cm}$ from the array and can be considered to be in the far field.

geometry of Figure 3, with the largest dimension, D, of each ETSA of the order of $10 \mathrm{~cm}$, we find that for frequencies between 2 and $5.3 \mathrm{GHz}$, we are in the far-field while for frequencies between 5.3 and $8 \mathrm{GHz}$, we are in the radiating near-field region. Thus we use the complete vector for the infinitesimal dipole to include all the relevant terms.

Figure 4 shows a single transmission experiment with antenna A as the transmitter and antennas A through $\mathrm{H}$ as receivers. The scattered hyperbolic arrival from the target, located at $40 \mathrm{~cm}$ from the center of the array, is very clear and has been obtained by subtracting the background scattered field, as described previously. The arrivals are essentially pulses, due to the large bandwidth of $6 \mathrm{GHz}$. We note that antenna A, the transmitter, also acting as a receiver, has a spurious arrival near 0 ns, possibly due to the network analyzer calibration, since a separate circuit is used to obtain a response from an antenna that is used in transmission and reception. This artifact will be evident in all transmission experiments presented.

Shown to the right of the differentially processed data is the image obtained using the retro-propagation algorithm described earlier. At each candidate scattering point, the summed time-reversed, retropropagated data is squared then integrated over time, with the total integral assigned to the pixel representing the CSP. This energy image in normalized to the maximum and then converted to dB. Figure 4 is the image of the scatterer in the $z=0$ plane, 

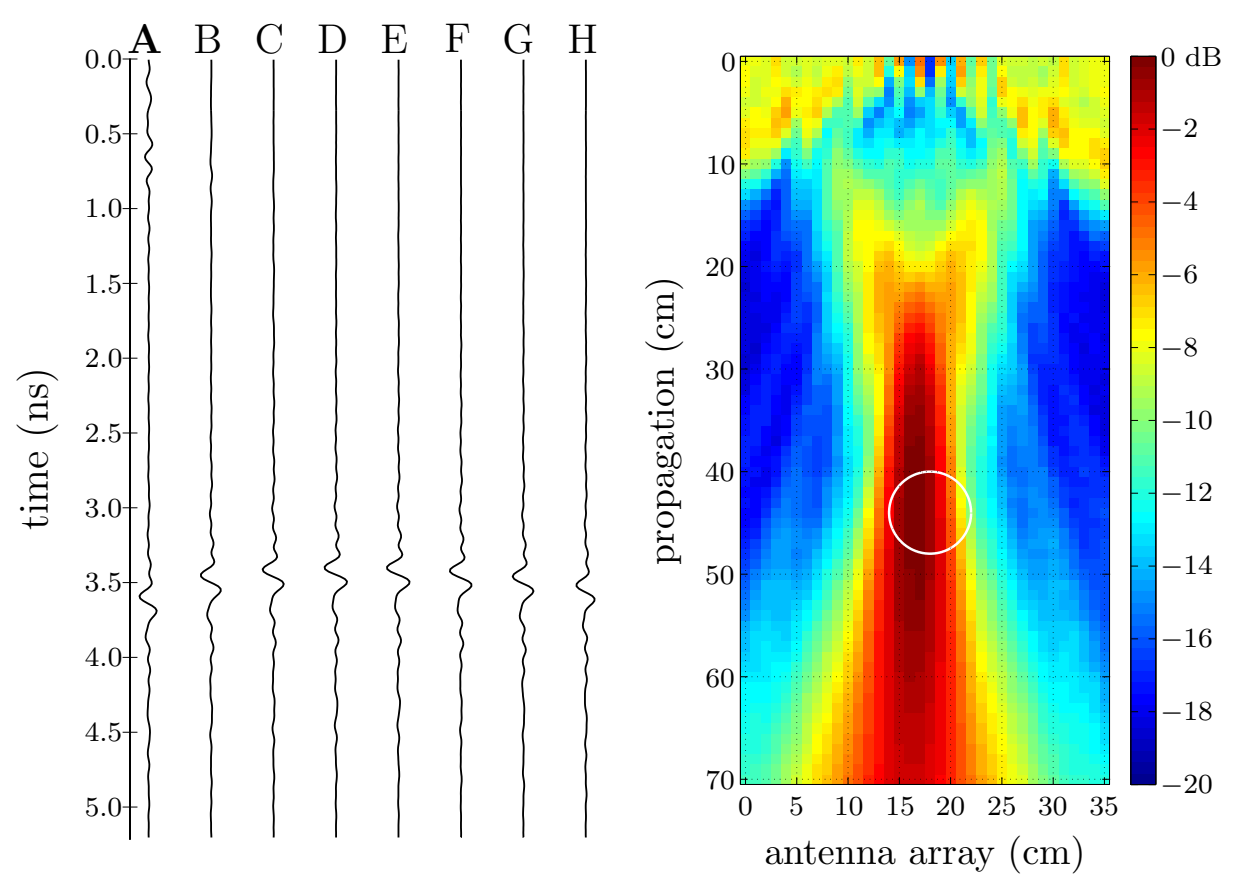

Fig. 4. Antenna A is the transmitter with scattered data, seen as a hyperbolic arrival, on the left, recorded by the whole array. On the right is the normalized image, expressed in $\mathrm{dB}$ relative to the maximum, with the white circle representing the bottle in the $z=0$ plane.

with the cross-section of the target shown by the white circle. The same data and image format are shown in Figure 5 through 11, sequentially presented to illustrate the data and image obtained, as each antenna functions as a transmitter, with the whole array receiving. A final image is shown in Figure 12. The image on the left was obtained by stacking the images obtained from the eight transmission experiments, scaling by the maximum value and converting to $\mathrm{dB}$ and the one on the right is $-3 \mathrm{~dB}$ threshold, meaning that all power values, less than half of the maximum, associated with a particular CSP, are assigned the deepest blue.

So far, we have presented images of the target at $z=0$. We expect that the method will work at other levels of $z$, since the target has limited extent in the $z$ direction $(z=0$ is in the middle of the target, which has a total height of $35 \mathrm{~cm}$ ). In Figure 13, six panels are presented that show the target image at increments of $5 \mathrm{~cm}$. The format of presentation is the same as Figure 12, normalized and thresholded at $-3 \mathrm{~dB}$. We would expect the target to disappear at $z=17.5 \mathrm{~cm}$. We note that indeed somewhere, between the $z=15$ and $z=20 \mathrm{~cm}$ panels, the target is much weaker, if we compare the normalized $\mathrm{dB}$ values. To obtain the images in this figure, the extra step of polarization projection was done, since the secondary sources and the receiving antennas, modeled as dipoles, are not co-planar. 

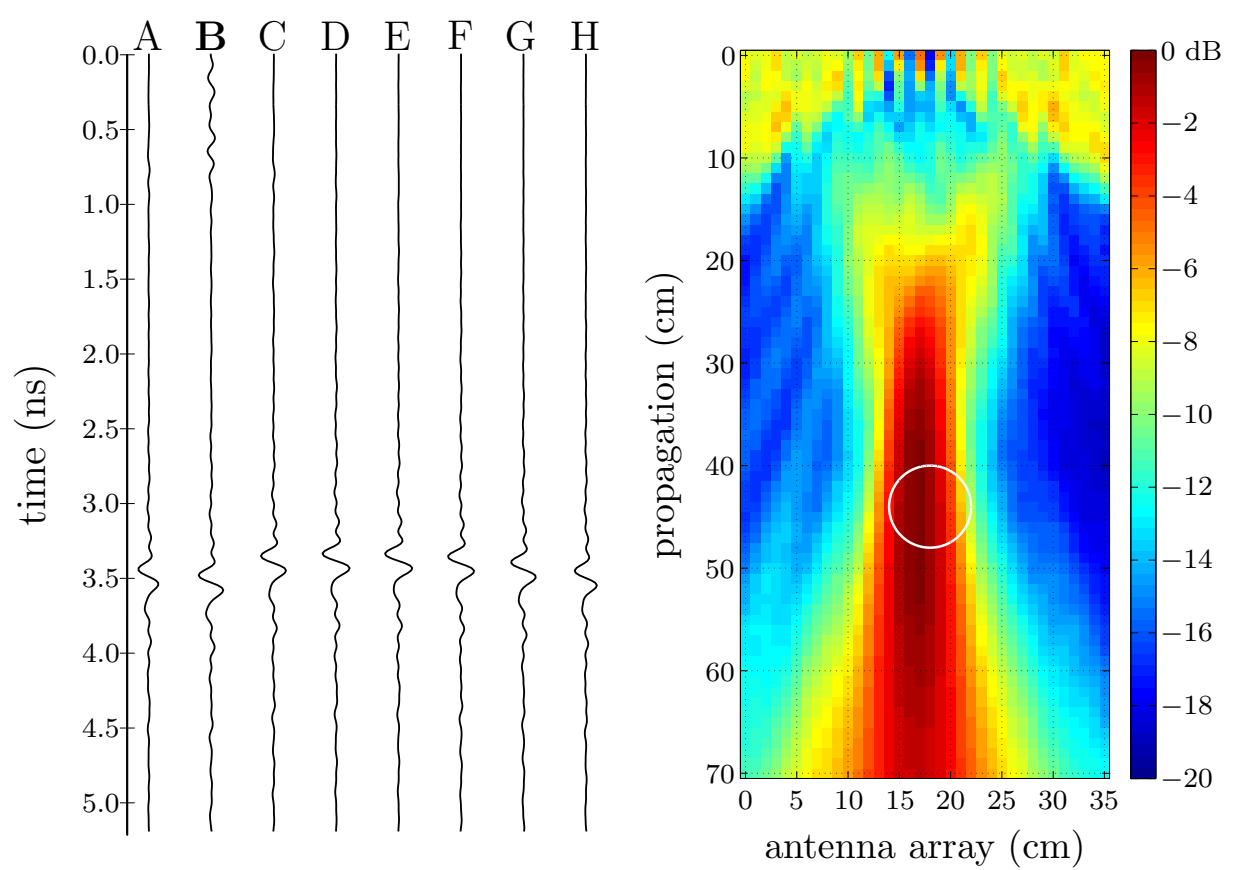

Fig. 5. Same images as in Fig. 4 with antenna B as transmitter.

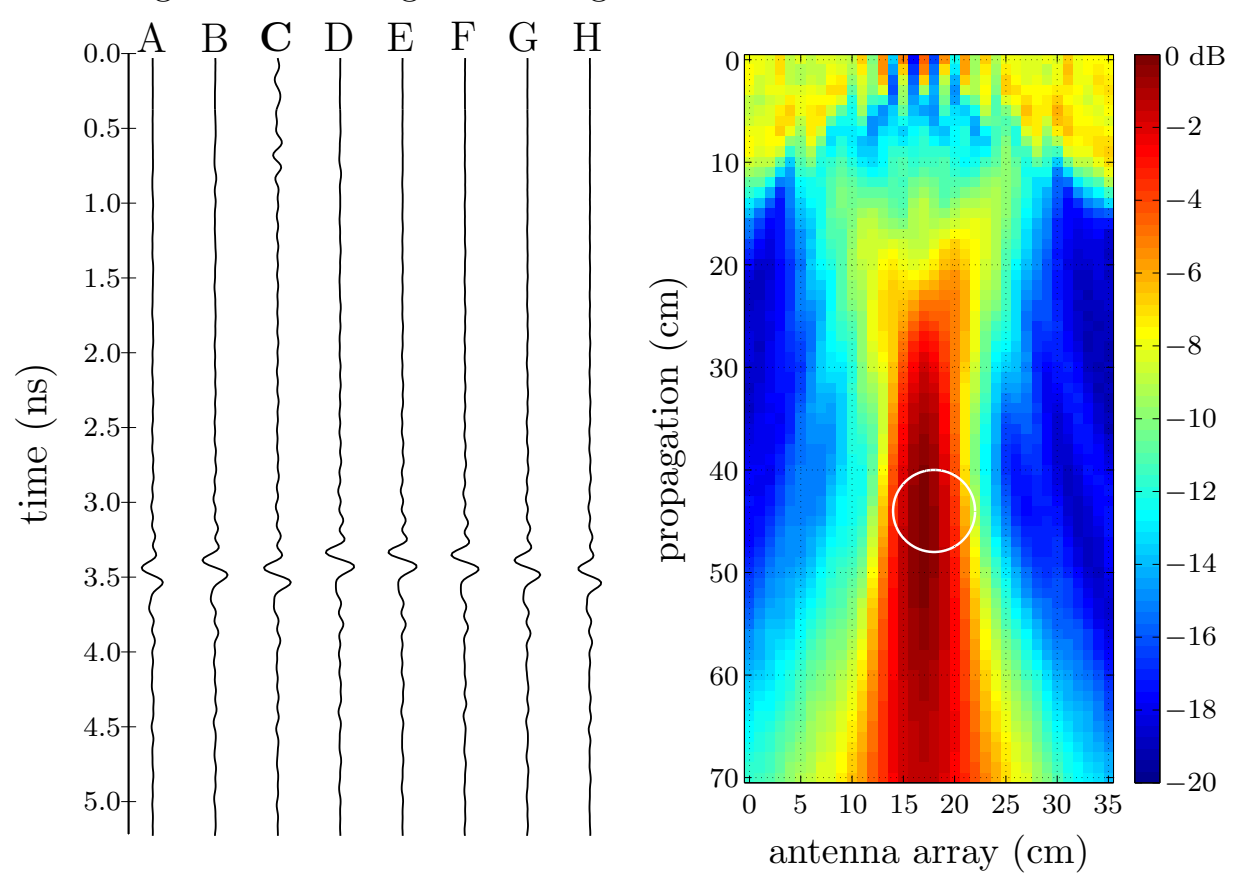

Fig. 6. Same images as in Fig. 4 with antenna $\mathrm{C}$ as transmitter.

\subsection{Retropopagation Movies}

In Figure 14, a sequence of twelve energy snapshots is shown, whose computation is based on the algorithm described in Subsection 2.3. The first snapshot in the sequence is Figure 14a while the snapshot corresponding to the best focus at the scatterer, located at a distance $40 \mathrm{~cm}$ from the front of the array and centered at $17.5 \mathrm{~cm}$, corresponds to Figure $14 \mathrm{j}$. 

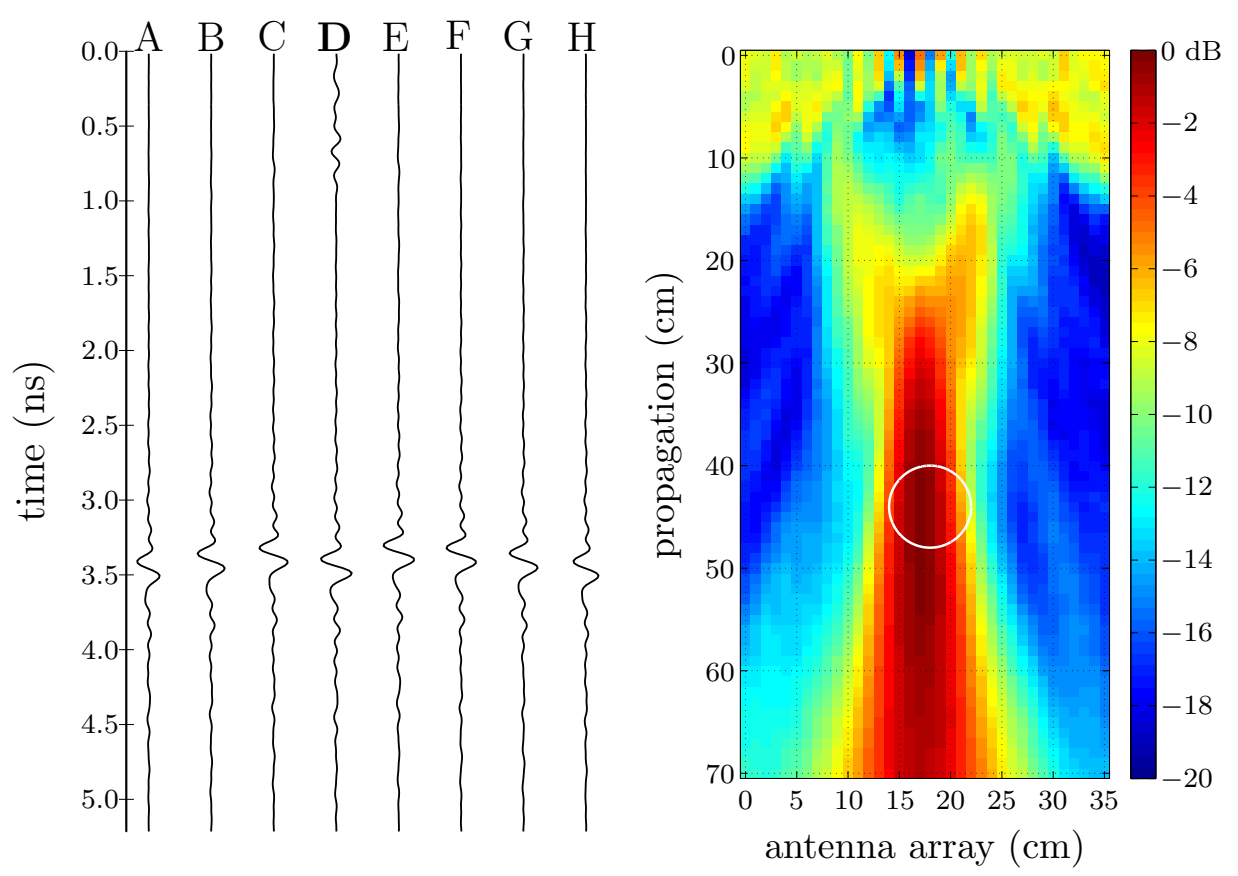

Fig. 7. Same images as in Fig. 4 with antenna D as transmitter.

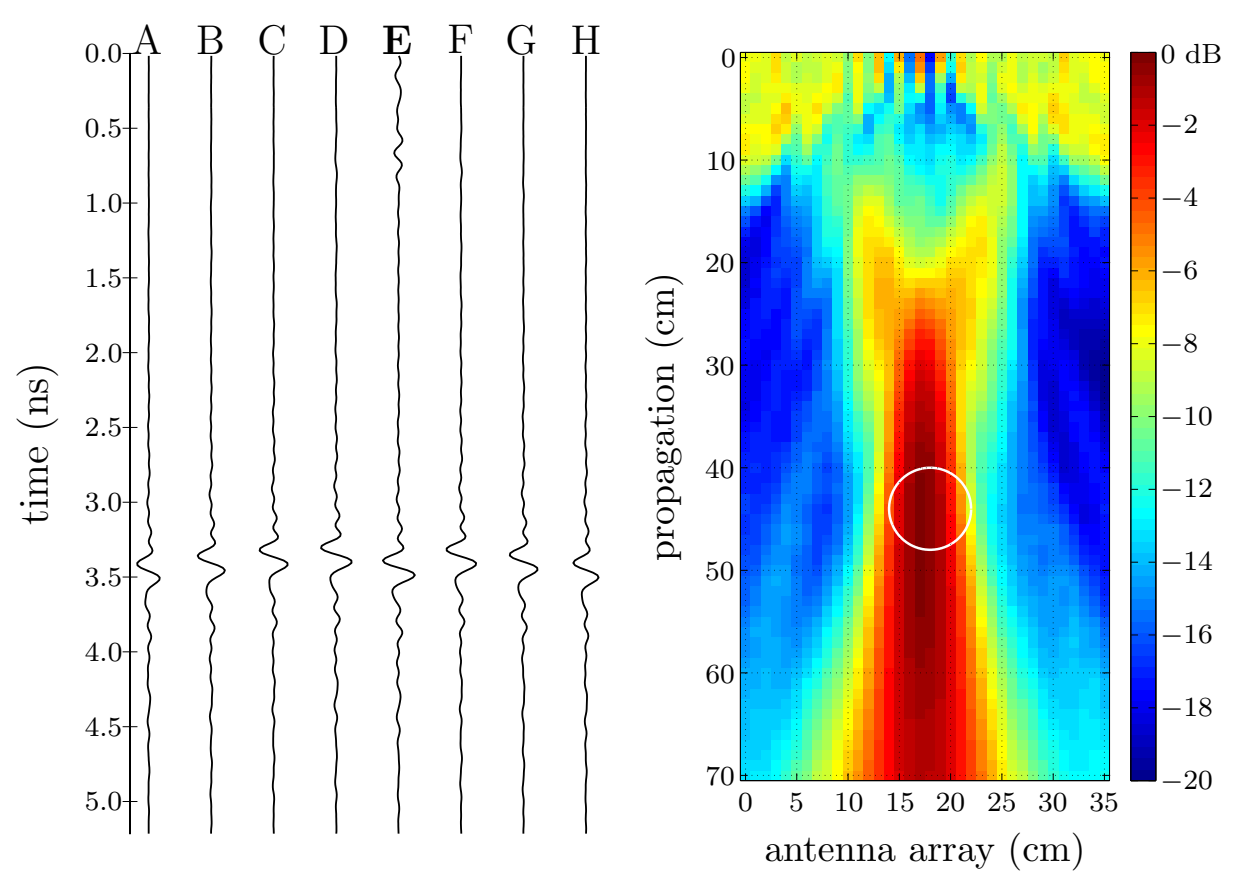

Fig. 8. Same images as in Fig. 4 with antenna E as transmitter.

A second sequence is shown in Figure 15, in which twelve energy snapshots are also presented, for the case of two targets. The first target is the same as that used in Figure 14 but located at a distance of $43 \mathrm{~cm}$ and at a lateral distance of $21 \mathrm{~cm}$, while the second target is a metallic cylinder of radius $2 \mathrm{~cm}$, located at $37.5 \mathrm{~cm}$ from the front of the array and at a lateral distance of $7.5 \mathrm{~cm}$. In this example, it is not as easy to pinpoint the snapshot corresponding to the best focus, due to the fact that the time for best focusing is not identical for 

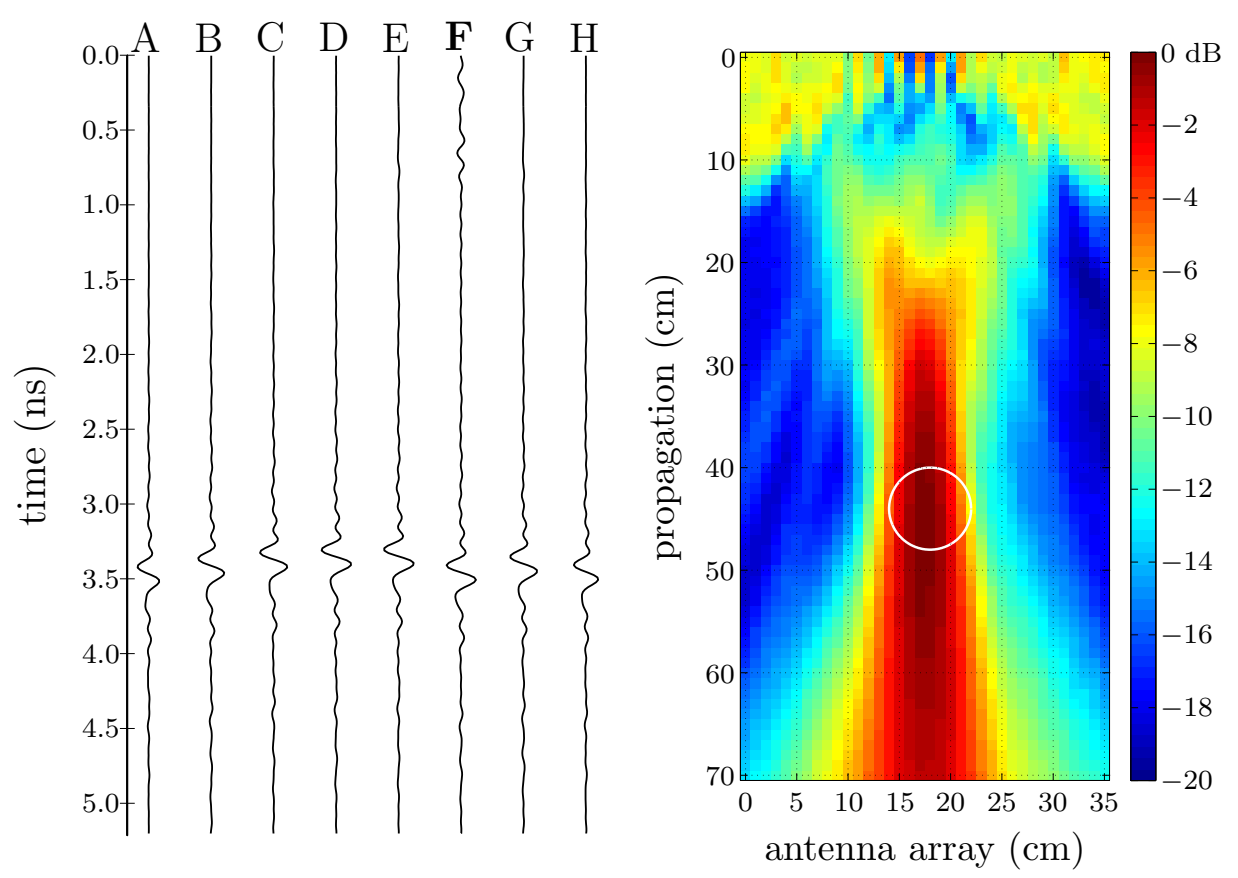

Fig. 9. Same images as in Fig. 4 with antenna $\mathrm{F}$ as transmitter.

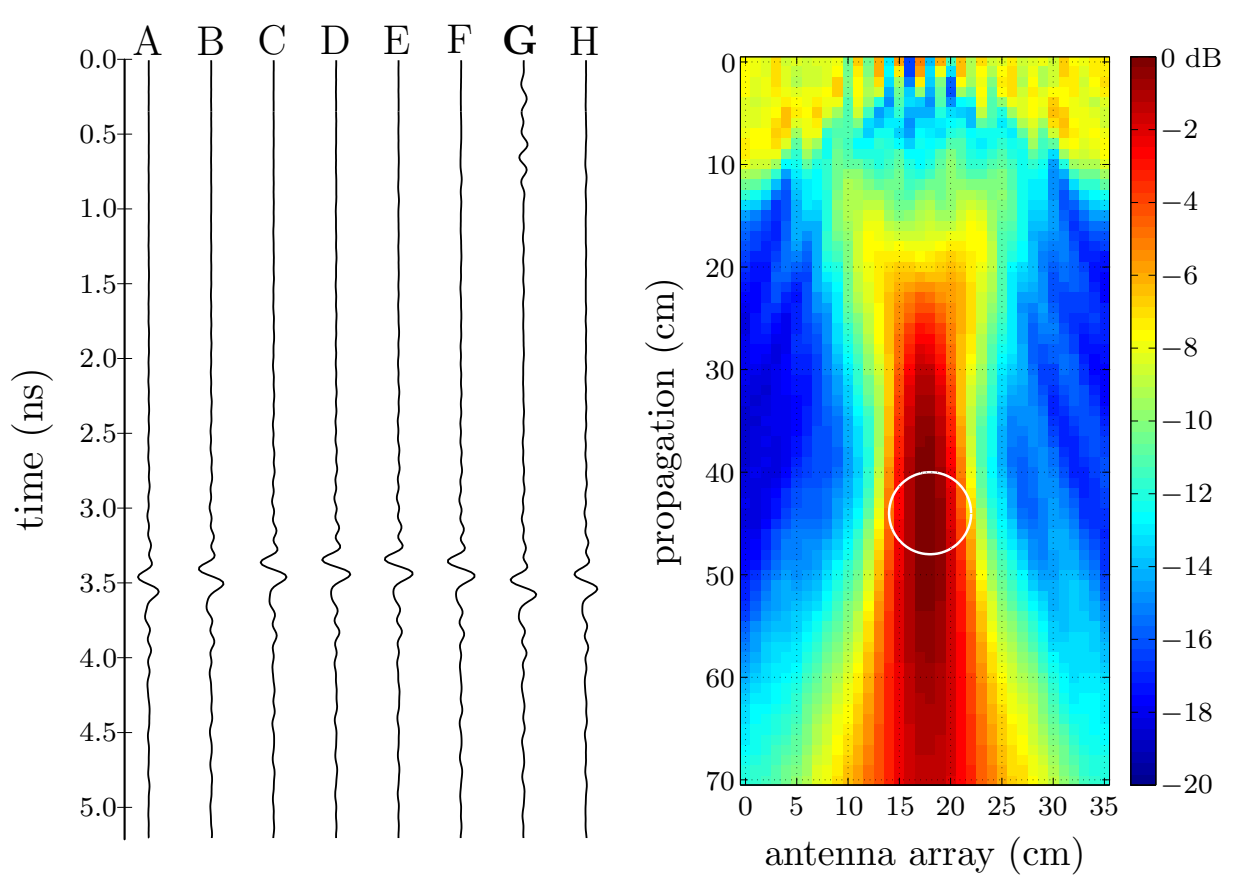

Fig. 10. Same images as in Fig. 4 with antenna $G$ as transmitter.

the two targets. A better focusing criterion will be the center of attention for future work. 

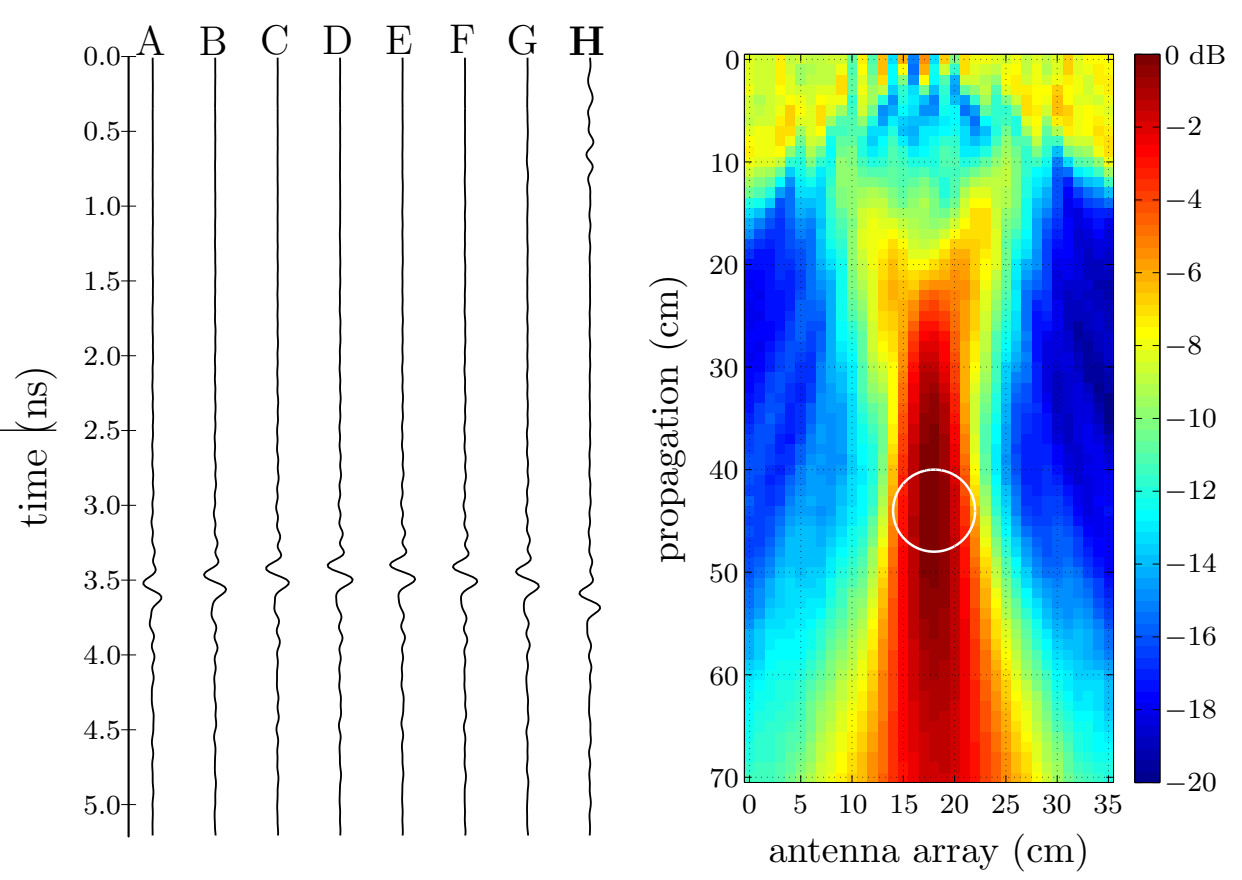

Fig. 11. Same images as in Fig. 4 with antenna $\mathrm{H}$ as transmitter.
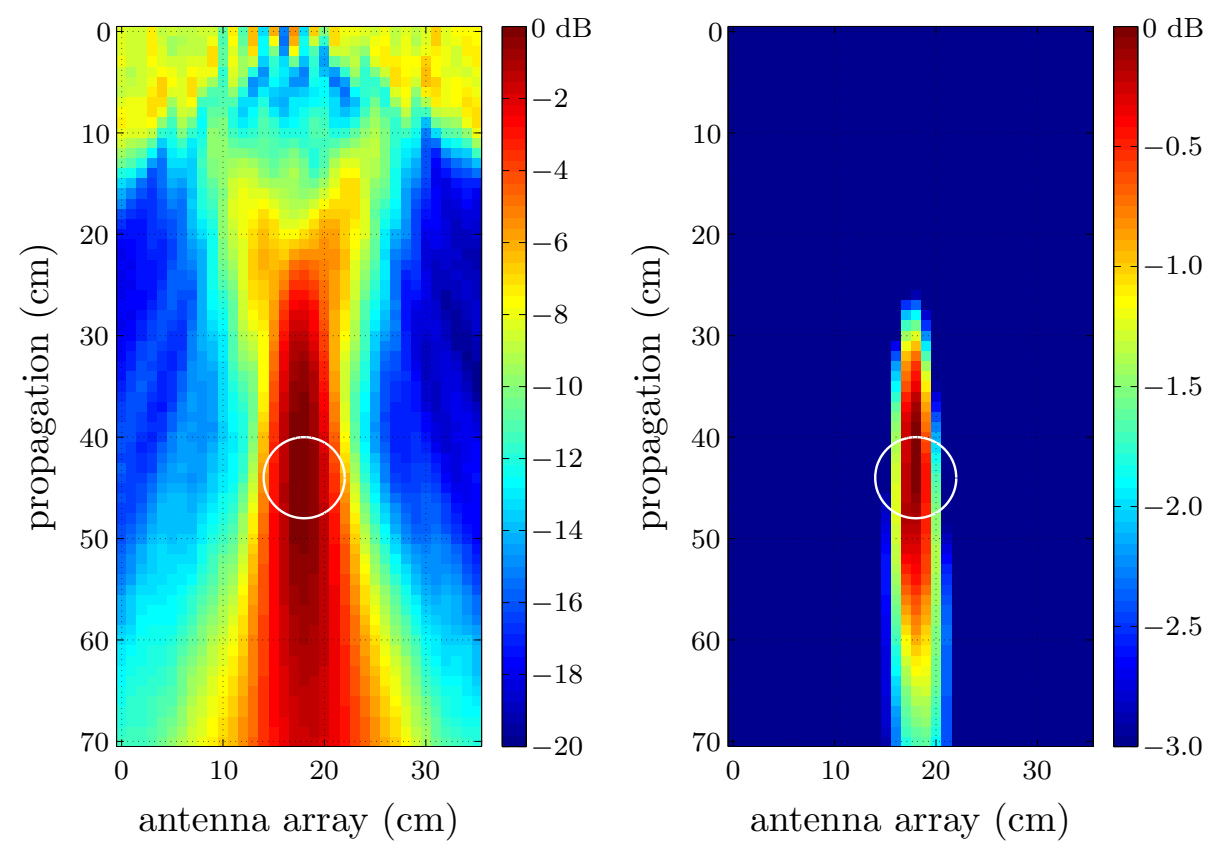

Fig. 12. Stacked images, at $z=0$ of the previous eight experiments, normalized and converted to $\mathrm{dB}$. The one on the left is a full scale image whereas the other one is a $-3 \mathrm{~dB}$ threshold image.

\section{Discussion}

The results of Section 3.1 clearly show promise in imaging scatterers in an exterior experiment, that is, when an isolated scatterer can be illuminated using a UWB array. The images obtained are blurred due to aperture effects 


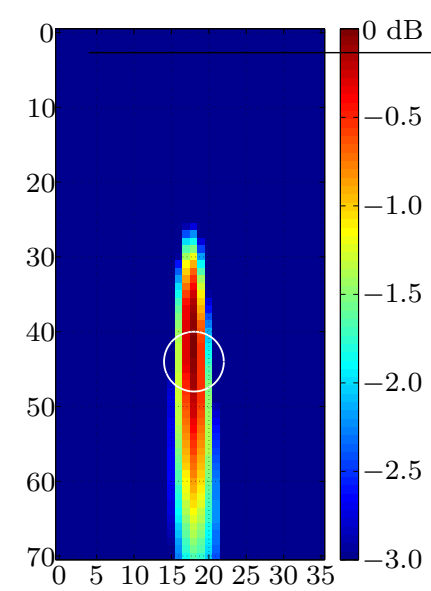

(a)

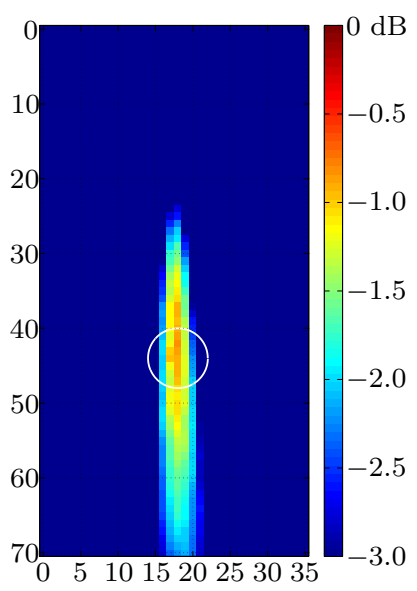

(d)

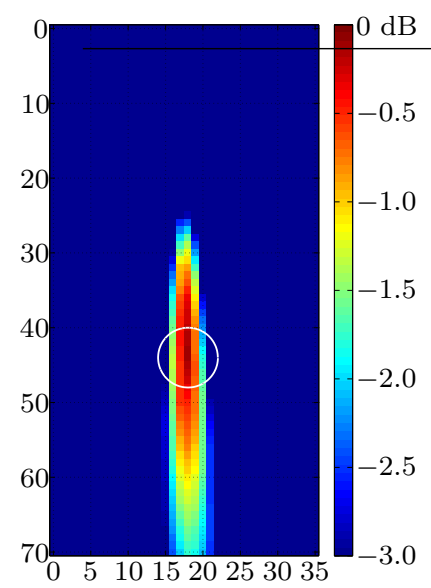

(b)

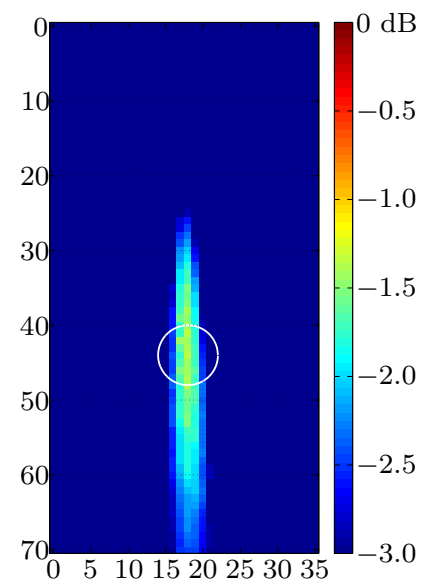

(e)

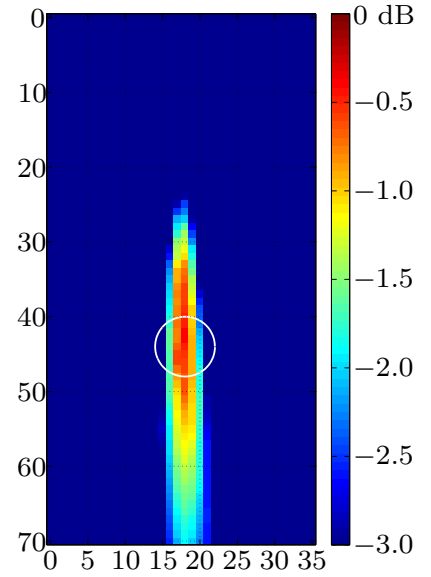

(c)

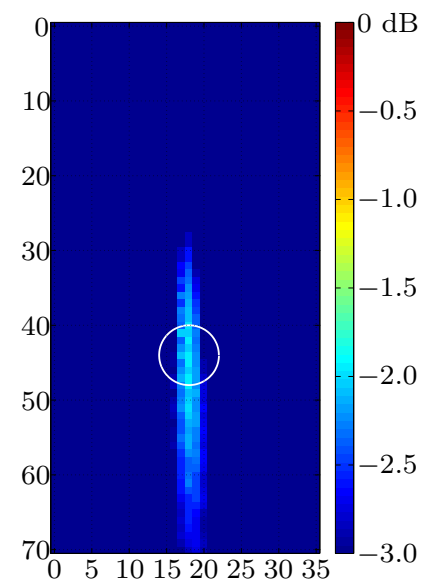

(f)

Fig. 13. Stacked images, at different $z$ levels, of the eight target illuminations, normalized and converted to $\mathrm{dB}$, with a $-3 \mathrm{~dB}$ threshold scale; a) $z=5 \mathrm{~cm}$; b) $z=10 \mathrm{~cm}$; ) $z=15 \mathrm{~cm}$; d) $z=20 \mathrm{~cm}$; e) $z=25 \mathrm{~cm}$; f) $z=30 \mathrm{~cm}$. The horizontal axis corresponds to the antenna array and the vertical one to the propagation distance, both in centimeters.

and the limited bandwidth of $6 \mathrm{GHz}$. More resolved images can be obtained by using a wider bandwidth and by employing a larger array size, an effort that is already underway at LEAT.

The reverse-time algorithm can also be improved using a more sophisticated model of the receiving antenna, which is currently modeled as an electrically small dipole. Despite the simplifications of the re-radiating process and the model for the antennas, initial images were obtained that approximately conform to the target.

The initial success of the employment of these high-frequency Vivaldi antennas indicate the promise of using a lower frequency antenna as an alternative 


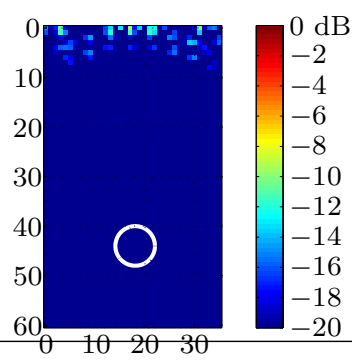

(a)

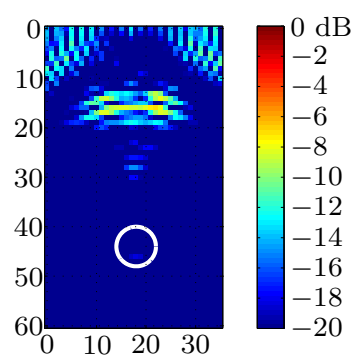

(d)

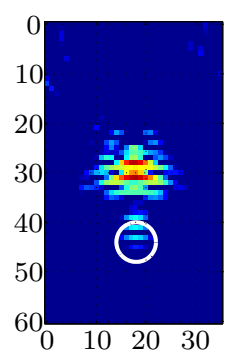

(g)

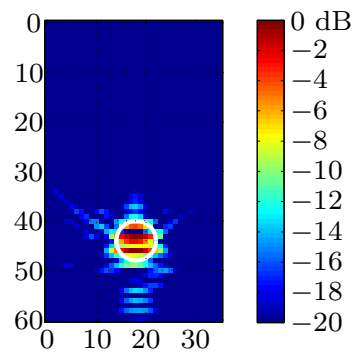

(j)

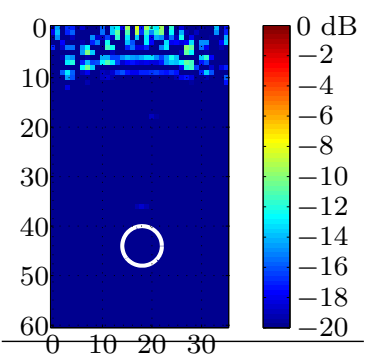

(b)

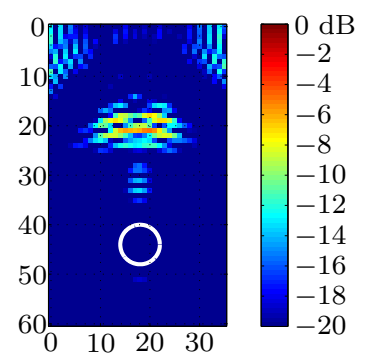

(e)

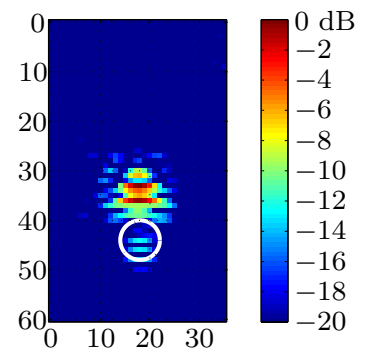

(h)

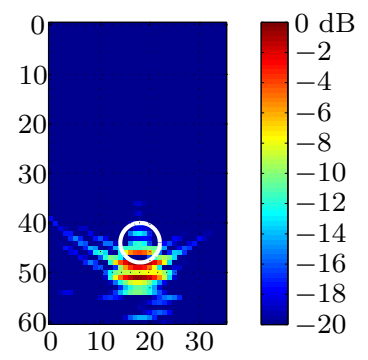

(k)

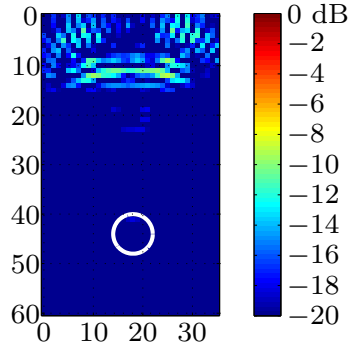

(c)

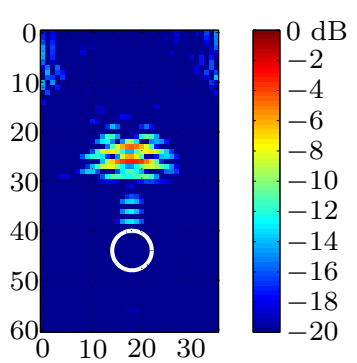

(f)

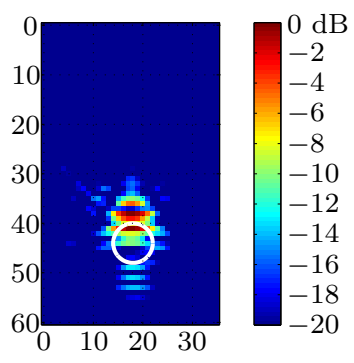

(i)

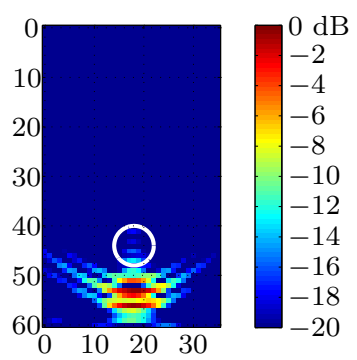

( $\ell$

Fig. 14. Twelve sequential snapshots, a) to l), of retropropagated scattered energy from a plastic bottle target, of radius $4 \mathrm{~cm}$, filled with saline. The target is located at $40 \mathrm{~cm}$ from the front of the antenna array and laterally at the array center. The horizontal axis corresponds to the antenna array and the vertical one to the propagation distance, both in centimeters. 


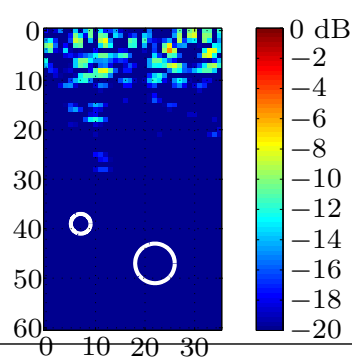

(a)

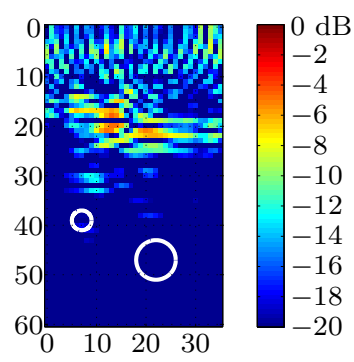

(d)

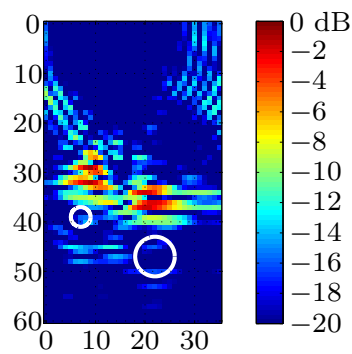

(g)

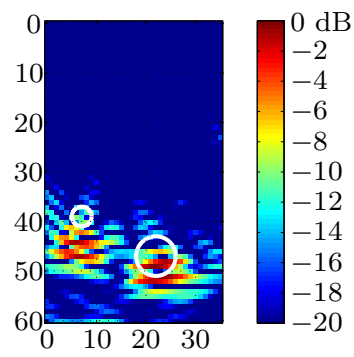

(j)

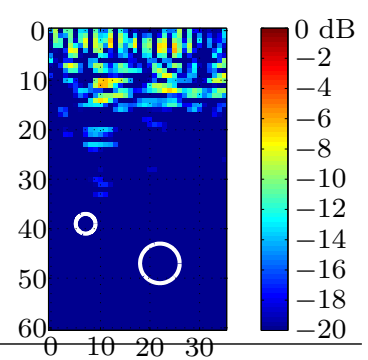

(b)

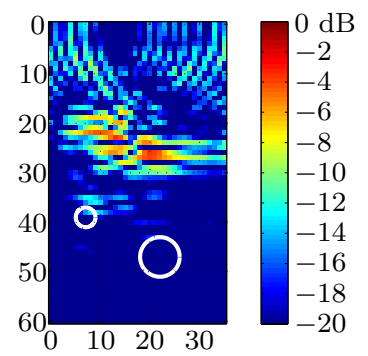

(e)

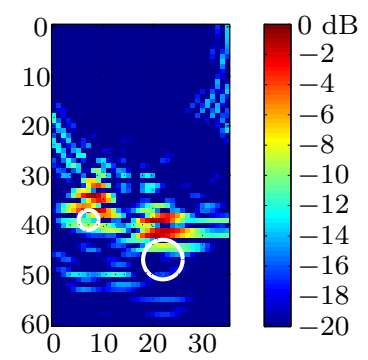

(h)

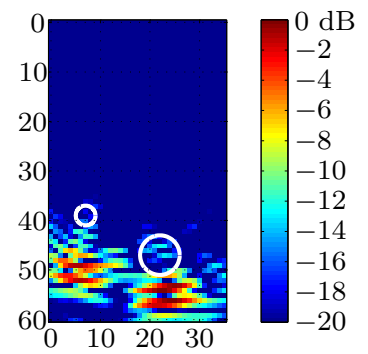

(k)

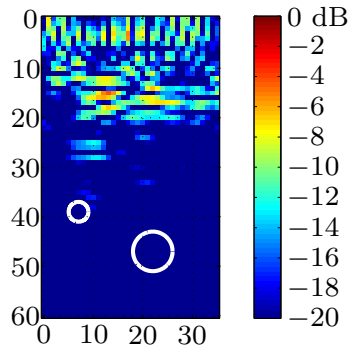

(c)

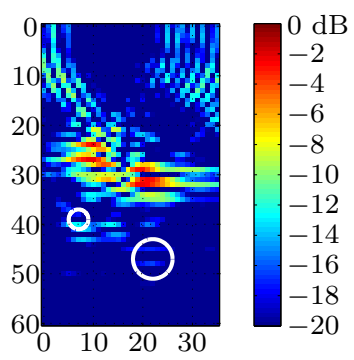

(f)

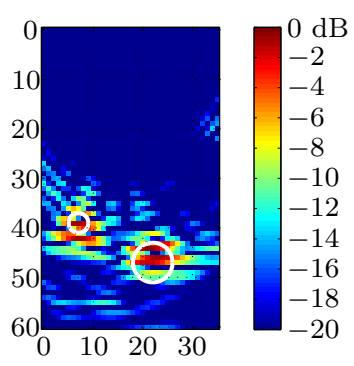

(i)

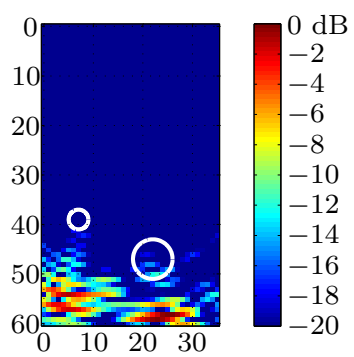

$(\ell)$

Fig. 15. Twelve snapshots of the energy retropropagated as a function of reverse time for the two-target case, consisting of a plastic bottle filled with saline and an adjacent metal cylinder. The horizontal axis corresponds to the antenna array and the vertical one to the propagation distance, both in centimeters. 


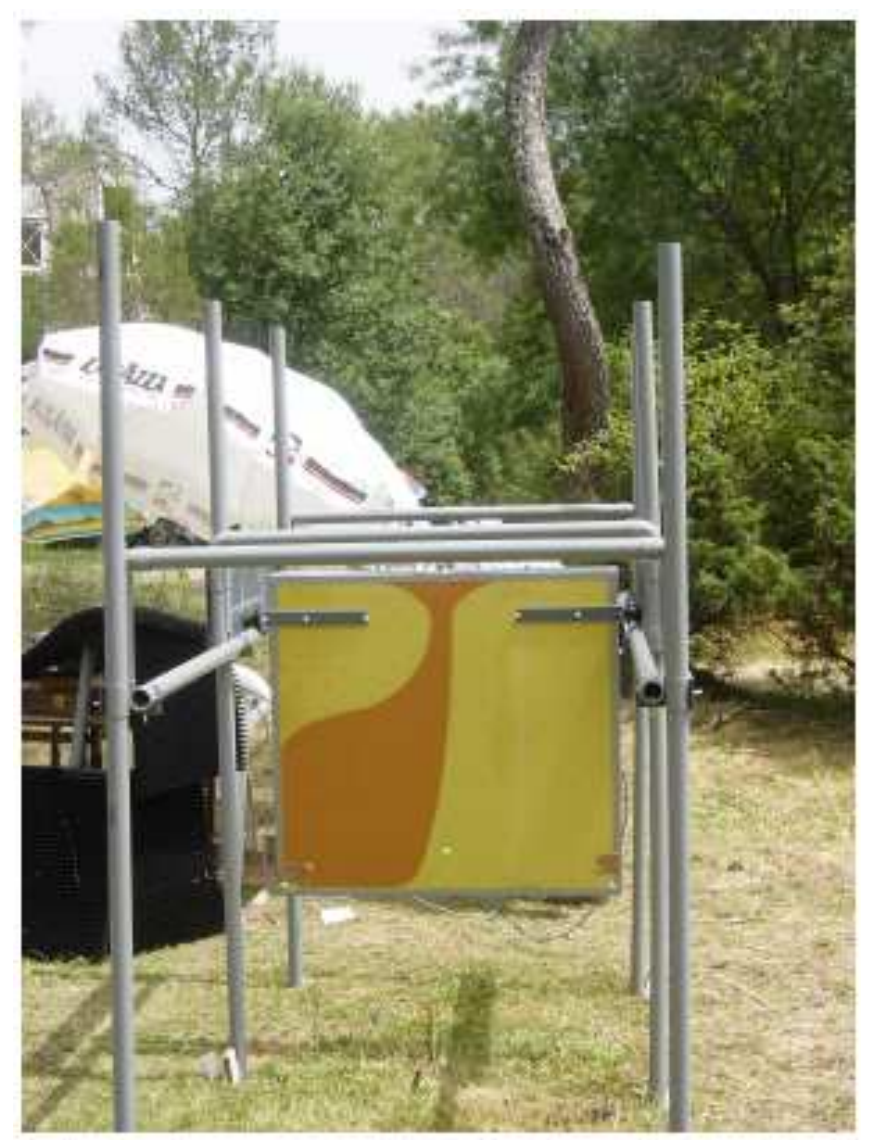

Fig. 16. View of the large-scale exponentially tapered slot antenna mounted on a carriage for test data collection.

to conventional GPR antennas. We have constructed such an antenna, shown in Figure 16. The bandwidth of this antenna is from $120 \mathrm{MHz}$ to $2.5 \mathrm{GHz}$ and offers the possibility of obtaining very clean data, at greater depths, without the conventional ringing and necessary pre-processing step of predictive deconvolution to eliminate this ringing.

\section{Conclusions}

We have shown that the application of a time-reversed imaging algorithm, applied to UWB differential array data measurements, can be successfully employed to obtain target images for an exterior scattering problem. The derived images from the data employed an elementary dipole model for the ensemble of secondary radiators, and the same dipole model for the receiving antenna. The images obtained conformed approximately to the target location. Two retropropagation sequences were presented, demonstrating the efficacy of using the time-reversal technique, applied in the context of electromagnetic scattering. An important extension to this work will be the development of 
an appropriate stopping condition, in order to obtain the image with the best focus.

\section{Acknowledgments}

The authors would like to thank Jean-Louis Le Sonn, Laurent Brochier and Franck Perret for making the data collection possible.

\section{References}

Baysal, E., Kosloff, D., Sherwood, J., 1983. Reverse time migration. Geophysics 48, 1514.

Bolomey, J.-C., Izadnegahdar, A., Jofre, L., Pichot, C., Peronnet, G., Solaimani, M., November 1982. Microwave Diffraction Tomography for Biomedical Applications. IEEE Transactions on Microwave Theory and Techniques 30 (11), 1998-2000.

Caorsi, S., Massa, A., Pastorino, M., Donelli, M., June 2004. Improved microwave imaging procedure for nondestructive evaluations of twodimensional structures. IEEE Transactions on Antennas and Propagation 52 (6), 1386-1397.

Chang, W., McMechan, G., 1987. Elastic reverse-time migration. Geophysics $52,1365$.

Chang, W., McMechan, G., 1990. 3D acoustic prestack reverse-time migration 1. Geophysical Prospecting 38 (7), 737-755.

Chatelée, V., December 2006. Développement d'un système d'imagerie microonde multistatique ultra large bande. Application à la détection d'objets en régime temporel et fréquentiel. Ph.D. thesis, Université de Nice Sophia Antipolis.

Daniels, D. J., 2004. Ground Penetrating Radar, 2nd Edition. No. 15 in IEE Radar, Sonar, Navigation and Avionics Series. The Institution of Electrical Engineers, London.

Derode, A., Tourin, A., Fink, M., May 2002. Time reversal versus phase conjugation in a multiple scattering environment. Ultrasonics 40 (1-8), 275-280.

Fink, M., July-August 2006. Time-reversal acoustics in complex environments. Geophysics 71 (4), SI151-SI164.

Fink, M., Cassereau, D., Derode, A., Prada, C., Roux, P., Tanter, M., Thomas, J.-L., Wu, F., December 2000. Time-reversed acoustics. Reports on Progress in Physics 63 (12), 1933-1995.

Gibson, P., September 17-21, 1979. The Vivaldi aerial. In: Proceedings of the 9th European Microwave Conference. Brighton, UK, pp. 101-105.

Guillanton, E., December 2000. Étude d'un système d'imagerie microonde 
multistatique-multifréquence pour la reconstruction d'objets enfouis. Ph.D. thesis, Université de Nice Sophia Antipolis.

Guillanton, E., Dauvignac, J.-Y., Pichot, C., Cashman, J., November 1998. A new design tapered slot antenna for ultra-wideband applications. Microwave and Optical Technology Letters 19 (4), 286-289.

Holmes, J. F., Jacques, S. L., Hunt, J. M., 2000. Adapting atmospheric LIDAR techniques to imaging biological tissue. In: Proceedings of SPIE the International Society for Optical Engineering. Vol. 3927. pp. 226-231.

Johnson, R. H., Poeter, E. P., 2003. Interpreting DNAPL saturations in a laboratory-scale injection with GPR data and direct core measurements. Tech. rep., U.S. Geological Survey.

URL http://pubs.usgs.gov/of/2003/ofr-03-349/

Marklein, R., Mayer, K., Hannemann, R., Krylow, T., Balasubramanian, K., Langenberg, K., Schmitz, V., December 2002. Linear and nonlinear inversion algorithms applied in nondestructive evaluation. Inverse Problems 18 (6), $1733-1759$.

Miyakawa, M., Ishida, T., Watanabe, M., September 1-5, 2004. Imaging capability of an early stage breast tumor by CP-MCT. In: Proceedings of the 27th Annual International Conference of the IEEE Engineering in Medicine and Biology Society (IEMBS 2004). Vol. 2. pp. 1427-1430.

Miyakawa, M., Orikasa, K., Ishi, N., Bertero, M., Boccacci, P., October 1999. Numerical analysis of CP-MCT and image restoration by the computed projection. Vol. 2. p. 1109.

Munk, J., Sheets, R., 1997. Detection of underground voids in Ohio by use of geophysical methods. Tech. rep., U.S. Geological Survey.

Pastorino, M., Caorsi, S., Massa, A., May 21-23; 2001. A global optimization technique for microwave nondestructive evaluation. In: Proceedings of the 18th IEEE Instrumentation and Measurement Technology Conference (imtc 2001). Vol. 3. pp. 1916-1920.

Pichot, C., Dauvignac, J.-Y., Aliferis, I., Le Brusq, E., Ferrayé, R., Chatelée, V., May 14, 2004. Recent Nonlinear Inversion Methods and Measurement System for Microwave Imaging. In: Proceedings of the IEEE International Workshop on Imaging Systems and Techniques. Stresa, Italy, pp. 95-99.

Pichot, C., Jofre, L., Peronnet, G., Bolomey, J.-C., April 1985. Active Microwave Imaging of Inhomogeneous Bodies. IEEE Transactions on Antennas and Propagation 33 (4), 416-425.

Sneddon, K. W., Powers, M. H., Johnson, R. H., Poeter, E. P., 2002. Modeling GPR data to interpret porosity and DNAPL saturations for calibration of a 3-D multiphase flow simulation. Tech. rep., U.S. Geological Survey.

URL http://pubs.usgs.gov/of/2002/ofr-02-451/

Wapenaar, K., Fokkema, J., Snieder, R., 2005. Retrieving the Green's function in an open system by cross correlation: A comparison of approaches (L). The Journal of the Acoustical Society of America 118, 2783.

Yarovoy, A., Ligthart, L. P., Schukin, A., Kaploun, I., May 2003. Fullpolarimetric video impulse radar for landmine detection: experimental ver- 
ification of main ideas. In: Proceedings of the 2nd International Workshop on Advanced GPR. Delft, Netherlands, pp. 148-155. 